\title{
EDITORIAL
}

\section{Precision noninvasive brain stimulation: is it precise? Is it needed?}

\author{
Lucas Borrione, ${ }^{1,2}$ Andre R. Brunoni ${ }^{1,2,3,4}$ iD \\ ${ }^{1}$ Departamento e Instituto de Psiquiatria, Faculdade de Medicina, Universidade de São Paulo (USP), São Paulo, SP, Brazil. ${ }^{2}$ Laboratório de \\ Neurociências (LIM27), Instituto Nacional de Biomarcadores em Neuropsiquiatria (INBioN), USP, São Paulo, SP, Brazil. ${ }^{3}$ Departamento de \\ Clínica Médica, Faculdade de Medicina, USP, São Paulo, SP, Brazil. ${ }^{4}$ Hospital Universitário, USP, São Paulo, SP, Brazil.
}

Navigare necesse est, vivere non est necesse

(To navigate is necessary, to live is not)

Pompey the Great, circa 60 b.C.

Portuguese poet Fernando Pessoa, when translating this famous quote from Pompey, conferred it a unique interpretation by using the word "preciso" - which means both "precise" and "needed" - in his verses. Fast forward 2,000 years. The emerging field of "precision psychiatry" proposes that a multitude of data can be used to match subgroups of patients with treatment interventions to improve treatment outcomes and reduce adverse effects. ${ }^{1}$ This new conceptualization states that "one-size-fits-all" treatments will be soon surpassed by personalized interventions. But is precision psychiatry precise? Is it needed?

These questions resonate in the systematic review recently published in this journal. Marques et al. examined the results of six randomized controlled trials involving the use of repetitive transcranial magnetic stimulation (rTMS) on the medial prefrontal cortex (mPFC) for different psychiatric disorders. ${ }^{2}$ So-called "precision noninvasive brain stimulation (NIBS)" carries an added level of complexity: the spatial coil or electrode localization should be taken into account. In fact, rTMS is only moderately effective in depression, which has encouraged researchers to target areas other than the usual dorsolateral prefrontal cortex (DLPFC). Interest in mPFC thus represents a novel venue for TMS research in psychiatry, particularly for treatment of major depressive disorder. ${ }^{3}$

From a wider scientific perspective, identifying different target areas for rTMS strengthens a new paradigm in psychiatry, based on neurobiological diagnostic precision outcomes and the unveiling of biomarkers to tailor treatment strategies. ${ }^{1}$ This newborn precision psychiatry no longer relies solely on a set of clinical information gathered in a diagnostic interview, but also on information from other research domains (i.e., molecular biosignatures, big data, neurocircuits, and the omics). ${ }^{1}$ For instance, Drysdale et al. used resting-state connectivity from depressed patients to identify four "biotype" patterns, and then showed that two

Correspondence: André R. Brunoni, Serviço Interdisciplinar de Neuromodulação, Instituto de Psiquiatria, Rua Dr. Ovidio Pires de Campos, $785,2^{\circ}$ andar, Ala Sul, CEP 05403-000, São Paulo, SP, Brazil.

E-mail: brunoni@usp.br of them were associated with higher rates of response to rTMS over the dorsomedial prefrontal cortex (>60\%), whereas two were not $(<25 \%)$. These findings are promising, although still need to be prospectively replicated in placebo-controlled trials. ${ }^{4}$

The neurobiological rationale for the review article in question is based on the integration of the MPFC with large-scale brain networks, such as the default-mode network (DMN) and the salience network. ${ }^{5}$ In this sense, a little more explanation about the normal functioning of the DMN and the salience network, as well as their alterations in certain psychiatric disorders, is in order. Normal connectivity between DMN subregions (mPFC, posterior cingulate cortex, and angular gyrus) is observed during task-free conditions, when participants are asked to rest and remain introspective. ${ }^{6}$ In depression, on the other hand, restingstate analyses have shown overactivation of the DMN, with associated maladaptive rumination. ${ }^{6}$ The salience network, in turn, is composed of two subsystems, one of which links the mPFC with the amygdala, and the other with the striatum. ${ }^{2}$ This system normally detects "salient" changes in the environment and appraisal/expression of emotions. ${ }^{6}$ Its dysfunction has been associated with both anxious avoidance and anhedonia. ${ }^{2}$ Interestingly, Williams suggests TMS for patients with DMN dysfunction, and deep brain stimulation (DBS) for patients with salience network dysfunction, ${ }^{6}$ in line with the precision psychiatry paradigm.

Another important point in the review by Marques et al. is the evaluation of the different types of coils used in randomized controlled trials. Most of the reviewed trials used deep TMS (dTMS) coils, but in one trial with positive outcomes in obsessive-compulsive disorder and acrophobia, a superficial, circular coil was used instead. ${ }^{2}$ Headache and local discomfort were frequent, and one patient receiving a frequency of $20 \mathrm{~Hz}$ experienced a generalized (tonic-clonic) seizure. ${ }^{2}$ These adverse events are in line with current knowledge about TMS, which associates a greater risk of seizures to higher frequencies (especially $>10 \mathrm{~Hz}$ ) and the incidence of headache with the use of coils that reach deeper into the brain. Nonetheless, the

How to cite this article: Borrione L, Brunoni AR. Precision noninvasive brain stimulation: is it precise? Is it needed? Braz $\mathrm{J}$ Psychiatry. 2019;41:376-377. http://dx.doi.org/10.1590/1516-44462019-4110 
mPFC has been much less investigated than DLPFC stimulation, and safety and tolerability aspects remain a concern.

The final aspect that deserves mention regards the review authors' conclusions. Despite significant differences between mPFC TMS treatment and placebo in patients with OCD and acrophobia, the results of the remaining trials (in substance use disorders, autism spectrum disorders, depression, and posttraumatic stress disorder) were only significant in within-group analyses. ${ }^{2}$ The authors consider that the main limitation of the evidence was the high risk of bias in the majority of the studies and differences in landmarks for coil positioning, which made it likely that different divisions of the MPFC were stimulated in each study. ${ }^{2}$ More studies with better methods are needed in order to confirm the preliminary clinical findings of MPFC TMS.

The combination of TMS-related research with advances in functional neuroimaging will surely assist in choosing differential brain targets linked with mental disorders, with likely improvement of clinical outcomes. In this sense, the review by Marques et al. evokes the need for further development of the emerging field of precision NIBS, which is still not as precise as needed for a paradigm shift.

\section{Disclosure}

The authors report no conflicts of interest.

\section{References}

1 Fernandes BS, Williams LM, Steiner J, Leboyer M, Carvalho AF, Berk M. The new field of 'precision psychiatry'. BMC Med. 2017; 15:80.

2 Marques RC, Vieira L, Marques D, Cantilino A. Transcranial magnetic stimulation of the medial prefrontal cortex for psychiatric disorders: a systematic review. Braz J Psychiatry. 2019;41:447-57.

3 Brunoni AR, Sampaio-Junior B, Moffa AH, Aparício LV, Gordon P, Klein I, et al. Noninvasive brain stimulation in psychiatric disorders: a primer. Braz J Psychiatry. 2019;41:70-81.

4 Drysdale AT, Grosenick L, Downar J, Dunlop K, Mansouri FMeng Y, et al. Erratum: Resting-state connectivity biomarkers define neurophysiological subtypes of depression. Nat Med. 2017;23:264.

5 Menon V. Large-scale brain networks and psychopathology: a unifying triple network model. Trends Cogn Sci. 2011;15:483-506.

6 Williams LM. Precision psychiatry: a neural circuit taxonomy for depression and anxiety. Lancet Psychiatry. 2016;3:472-80. 